\title{
Rendimiento productivo y calidad de huevo en gallinas ponedoras alimentadas con torta de palmiste (Elaeis guineensis) y enzimas $\beta$-glucanasa y xilanasa
}

\author{
Productive performance and egg quality in laying hens feed palm kernel cake \\ (Elaeis guineensis) and enzymes $\beta$-glucanase and xylanase \\ José Cadillo C. ${ }^{1,3}$, Marcial Cumpa G. ${ }^{1}$, José Galarza F. ${ }^{2}$
}

\section{Resumen}

El objetivo del presente experimento fue evaluar el efecto de tres niveles de torta de palmiste $(0,3$ y $6 \%$ ) y la inclusión de dos niveles de enzimas $\beta$-glucanasa y xilanasa ( 0 y $0.05 \%$ ) sobre el desempeño productivo y la calidad de huevos en gallinas. Se utilizaron 216 gallinas de postura de la línea Hy-Line Brown de 46 semanas de edad y distribuidas en un diseño de bloques completamente al azar con un arreglo factorial de $3 \times 2$. El experimento duró 12 semanas. La inclusión de 3\% de torta de palmiste sin suplementación enzimática (T3) mejoró significativamente $(\mathrm{p}<0.05)$ el peso promedio del huevo comparado con el tratamiento control (T1). No hubo diferencias significativas entre tratamientos con respecto a peso inicial, peso final, ganancia de peso, consumo de alimento, conversión alimenticia, producción de huevos, masa de huevo, pigmentación y altura de yema, espesor de cascara, altura de clara y unidades Haugh, asi como en los consumos de energía, proteína, lisina y metionina + cistina.

Palabras clave: enzimas; huevo; gallinas; producción; torta palmiste

\section{AbStRaCT}

The aim of this experiment was to evaluate the effect of three levels of palm kernel cake $(0,3$ and $6 \%)$ and the inclusion of two levels of enzymes $\beta$-glucanase and xylanase $(0$ and $0.05 \%)$ on the productive performance and quality of eggs in hens. Laying hens of the Hy-Line Brown line $(n=216)$ of 46 weeks of age were used and distributed in a

\footnotetext{
${ }^{1}$ Unidad Experimental de Avicultura, Programa de Investigación y Proyección Social en Aves, Facultad de Zootecnia, Universidad Nacional Agraria La Molina, Lima, Perú

${ }^{2}$ Escuela de Post Grado, Universidad Nacional Agraria La Molina, Lima, Perú

${ }^{3}$ E-mail: jcadillo@lamolina.edu.pe
}

Recibido: 21 de agosto de 2018

Aceptado para publicacion: 6 de febrero de 2019 
completely randomized block design with a factorial arrangement of $3 \times 2$. The experiment lasted 12 weeks. The inclusion of 3\% palm kernel cake without enzyme supplementation (T3) significantly improved $(\mathrm{p}<0.05)$ the mean egg weight compared to the control treatment (T1). There were no significant differences between treatments with respect to initial and final weight, body weight gain, feed intake, feed conversion, egg production, egg mass, yolk pigmentation and yolk height, shell thickness, egg white height and Haugh units, as well as of energy, protein, lysine and methionine + cystine consumption.

Key words: enzymes; egg; hens; production; palm kernel cake

\section{INTRODUCCIÓN}

La industria avícola es una actividad altamente demandante de maíz amarillo duro y torta de soya, insumos utilizados en gran proporción en la alimentación de las aves en el Perú, pero que son producidos en cantidades menores a la demanda, por lo que año tras año se importan cantidades cada vez mayores. Según el Minagri (2018), en 2017 se importó 3326485 t de maíz amarillo duro, lo cual fue $9.8 \%$ más que en 2016 , así como $1252100 \mathrm{t}$ de torta de soya, $2 \%$ más que en 2016.

La dependencia de las importaciones de estos insumos obliga a los productores a buscar sustitutos que puedan reemplazarlos, al menos parcialmente. Estos insumos deben ser producidos con regularidad durante todo el año, deben tener un valor nutricional aceptable, y tener un precio cercano al maíz o la torta de soya, entre otras características. Uno de estos insumos en el Perú es la torta de palmiste, subproducto de la industrialización de la palma aceitera (Elaeis guinneensis), planta tropical propia de climas cálidos que crecen en zonas por debajo de los $500 \mathrm{msnm}$. Es un cultivo perenne, tardío y de largo rendimiento, pudiendo durar más de 50 años. Según el Minagri (2016), el cultivo de palma aceitera se ha constituido como una de las actividades agrarias con mayor crecimiento en la Amazonía peruana en los últimos años (de 236000 t en 2006 a 618000 t en 2014 de racimo de frutas frescas).
La torta de palmiste es utilizada en la alimentación animal, tanto en rumiantes como en monográstricos (aves y cerdos) como fuente proteica y energética, por lo que puede reemplazar parcialmente, tanto al maíz como a la torta de soya. Según FEDNA (2015) tiene $16.7 \%$ de proteína, 2340 de EM, 8-10\% de grasa y $12-16 \%$ de fibra. Ha sido utilizada en la alimentación de gallinas de postura en la fase de producción con buenos resultados (Chong et al., 2008; Muñoz, 2013; García et al., 2016). Por lo expuesto, el objetivo del presente estudio fue evaluar los niveles de uso de torta de palmiste con y sin suplementación enzimática ( $\beta$-glucanasa y xilanasa) sobre el rendimiento productivo y calidad del huevo en gallinas ponedoras.

\section{Materiales y Métodos}

La presente investigación se llevó a cabo en la Unidad Experimental de Avicultura del Programa de Investigación y Proyección Social en Aves, Facultad de Zootecnia, Universidad Nacional Agraria La Molina, ubicada en Lima, Perú. El trabajo tuvo una duración de 12 semanas y la fase experimental se hizo entre febrero y abril de 2017. Las gallinas tuvieron un periodo de 20 días de adaptación previo a las dietas.

Se emplearon 216 gallinas ponedoras de 46 a 57 semanas de edad de la línea Hy Line Brown de huevo rosado, distribuidas al azar en seis tratamientos, con seis unidades expe- 
Cuadro 1. Análisis químico de la torta de palmiste

\begin{tabular}{lc}
\hline Análisis & Valor \\
\hline Humedad (\%) & 7.98 \\
Proteína Total (N x 6.25) & 16.12 \\
$(\%)$ & \\
Grasa (\%) & 7.94 \\
Fibra cruda (\%) & 16.87 \\
Ceniza (\%) & 3.83 \\
ELN (\%) & 50.26 \\
Energía metabolizable & 2,260 \\
(kcal/kg) & \\
\hline
\end{tabular}

Laboratorio de Evaluación Nutricional de Alimentos (LENA) UNALM

rimentales (jaulas) por tratamiento de seis gallinas cada una. Los productos evaluados fueron la torta de palmiste (subproducto del procesamiento del aceite de palma), cuya composición química se muestra en el Cuadro 1 , y un complejo enzimático comercial Rovabio ${ }^{\mathrm{TM}}$ Excel AP, compuesto por endo-1, 3(4) $\beta$-glucanasa N. ${ }^{\circ}$ EC 3.2.1.6 $(2000 \mathrm{AGL}$ unidades/g) y endo-1,4- $\beta$-xilanasa $N{ }^{\circ}$ EC 3.2.1.8 (22 000 unidades visco/g), para uso en la alimentación animal.

Se utilizaron seis dietas experimentales, formuladas al mínimo costo con el programa Mixit-2, según los requerimientos del NRC (1994). La composición porcentual y el valor nutricional calculado de las dietas experimentales se muestran en el Cuadro 2. Las dietas fueron preparadas semanalmente en la Planta de Alimentos del Programa de Investigación y Proyección en Aves, Facultad de Zootecnia, Universidad Nacional Agraria La Molina.

Los tratamientos fueron los siguientes: $\mathrm{T} 1$, dieta control, sin torta de palmiste y sin $\beta$-glucanasa y xilanasa; T2, dieta sin torta de palmiste y $0.05 \%$ de $\beta$-glucanasa y xilanasa; $\mathrm{T} 3$, dieta con $3 \%$ de torta de palmiste y $\sin \beta$ - glucanasa y xilanasa; $\mathrm{T} 4$, dieta con $3 \%$ de torta de palmiste y $0.05 \%$ de $\beta$-glucanasa y xilanasa; $\mathrm{T} 5$, dieta con $6 \%$ de torta de palmiste y $\sin \beta$-glucanasa y xilanasa y $\mathrm{T} 6$, dieta con $6 \%$ de torta de palmiste y $0.05 \%$ de $\beta$ glucanasa y xilanasa.

En el rendimiento productivo se evaluó el peso inicial, peso final, ganancia de peso, producción de huevos, peso promedio del huevo, masa de huevo (producción de huevos x peso promedio del huevo/100), consumo de alimento y conversión alimenticia (consumo de alimento [g] / masa de huevo [g]); y en la calidad de huevo se evaluó la pigmentación de la yema (Abanico colorímetro DSM)), espesor de la cáscara, altura de la clara, altura de yema (calibrador digital 0-200 mm/08", KAMASA/KM-448) y las unidades Haugh $\left(\mu \mathrm{H}=100 * \log \left(\mathrm{h}-1.7 \mathrm{~W}^{0.37}+7.6\right)\right)$. Para el pesaje de los huevos se utilizó una balanza Pocket Scale MH-200 (200 g; precisión: 0.01 g) y para el pesaje de las gallinas y el alimento se utilizó una balanza electrónica Excell AW-30 (capacidad: $30 \mathrm{~kg}$; sensibilidad: $1 \mathrm{~g}$ ).

Se empleó un diseño en bloques completamente al azar (DBCA), con arreglo factorial $3 \times 2$, con seis repeticiones por tratamiento. El análisis de varianza se hizo usando el procedimiento de modelos lineales generales (GLM) del software SAS v. 9.3. El control fue contrastado con cada uno de los tratamientos usando la prueba de Dunnett $(\mathrm{p}<0.05)$.

\section{Resultados y Discusión}

\section{Rendimiento Productivo}

\section{Peso final y ganancia de peso}

En el Cuadro 3 se muestra el peso inicial y final (g/ave) y las ganancias de peso (g/ave) obtenidos durante las 12 semanas que duró el experimento en los seis tratamientos. En ninguno de los tres parámetros evaluados hubo diferencias estadísticas $(\mathrm{p}<0.05)$ entre 
Cuadro 2. Composición y valor nutricional calculado de las dietas experimentales

\begin{tabular}{|c|c|c|c|c|c|c|}
\hline Ingredientes & $\mathrm{T} 1$ & $\mathrm{~T} 2$ & $\mathrm{~T} 3$ & $\mathrm{~T} 4$ & T5 & T6 \\
\hline Torta de palmiste & - & - & 3.00 & 3.00 & 6.00 & 6.00 \\
\hline Maíz & 62.88 & 62.88 & 57.6 & 57.54 & 56.89 & 56.91 \\
\hline Torta de soya & 21.59 & 21.59 & 21.52 & 21.53 & 18.68 & 18.53 \\
\hline Carbonato de calcio & 11.01 & 11.08 & 10.99 & 10.99 & 10.74 & 10.74 \\
\hline Fosfato dicálcico & 1.73 & 1.62 & 1.72 & 1.72 & 1.42 & 1.41 \\
\hline Harina de pescado & - & - & - & - & 1.15 & 1.24 \\
\hline Aceite de palma & 1.68 & 1.67 & 4.00 & 4.00 & 4.00 & 4.00 \\
\hline Sal & 0.37 & 0.37 & 0.37 & 0.37 & 0.31 & 0.31 \\
\hline DL - Metionina & 0.17 & 0.17 & 0.18 & 0.18 & 0.17 & 0.17 \\
\hline Cloruro de colina $60 \%$ & 0.10 & 0.10 & 0.10 & 0.10 & 0.10 & 0.10 \\
\hline Bicarbonato de sodio & 0.10 & 0.10 & 0.10 & 0.10 & 0.10 & 0.10 \\
\hline Premix Vit. y Min. & 0.10 & 0.10 & 0.10 & 0.10 & 0.10 & 0.10 \\
\hline L-Lisina - HCL & 0.03 & 0.03 & 0.04 & 0.04 & 0.07 & 0.07 \\
\hline Promotor Albac Z-B & 0.05 & 0.05 & 0.05 & 0.05 & 0.05 & 0.05 \\
\hline Antioxidante & 0.05 & 0.05 & 0.05 & 0.05 & 0.05 & 0.05 \\
\hline Fungistático & 0.05 & 0.05 & 0.05 & 0.05 & 0.05 & 0.05 \\
\hline Microsecuestrante & 0.05 & 0.05 & 0.05 & 0.05 & 0.05 & 0.05 \\
\hline Treonina & 0.02 & 0.02 & 0.06 & 0.06 & 0.05 & 0.05 \\
\hline Complejo enzimático & - & 0.05 & - & 0.05 & - & 0.05 \\
\hline Pigmentante & 0.02 & 0.02 & 0.02 & 0.02 & 0.02 & 0.02 \\
\hline Total & 100 & 100 & 100 & 100 & 100 & 100 \\
\hline \multicolumn{7}{|c|}{ Valor Nutricional Calculado (\%) } \\
\hline Proteína total & 14.71 & 14.71 & 14.70 & 14.70 & 14.70 & 14.70 \\
\hline E.M. (kcal/kg) & 2810 & 2810 & 2830 & 2830 & 2810 & 2810 \\
\hline Fibra cruda & 2.57 & 2.57 & 3.01 & 3.01 & 3.54 & 3.54 \\
\hline Lisina & 0.78 & 0.78 & 0.78 & 0.78 & 0.78 & 0.78 \\
\hline Metionina & 0.42 & 0.42 & 0.43 & 0.43 & 0.43 & 0.43 \\
\hline Metionina + cistina & 0.68 & 0.68 & 0.68 & 0.68 & 0.68 & 0.68 \\
\hline Fósforo disponible & 0.37 & 0.37 & 0.37 & 0.37 & 0.35 & 0.35 \\
\hline Calcio & 4.20 & 4.20 & 4.20 & 4.20 & 4.09 & 4.09 \\
\hline Sodio & 0.18 & 0.18 & 0.18 & 0.18 & 0.16 & 0.16 \\
\hline
\end{tabular}

T1, dieta control; T2, dieta sin torta de palmiste y $0.05 \%$ de $\beta$-glucanasa y xilanasa; T3, dieta con $3 \%$ de torta de palmiste y $\sin \beta$-glucanasa y xilanasa; T4, dieta con $3 \%$ de torta de palmiste y $0.05 \%$ de $\beta$-glucanasa y xilanasa; T5, dieta con $6 \%$ de torta de palmiste y $\sin \beta$-glucanasa y xilanasa y $\mathrm{T} 6$, dieta con $6 \%$ de torta de palmiste y $0.05 \%$ de $\beta$-glucanasa y xilanasa

los tratamientos, indicando que la inclusión de la torta de palmiste hasta $6 \%$, aún en ausencia de enzimas que favorecen la digestibilidad de la fibra, no afecta el peso de las gallinas de postura. Estos resultados problamente se deben a que los bajos niveles de inclusión de la torta de palmiste a la dieta, pese a su alto contenido de fibra, no afecta el contenido de fibra total de las dietas. 
Cuadro 3. Comportamiento productivo ${ }^{1}$ en gallinas ponedoras alimentadas con tres niveles de torta de palmiste y dos niveles de $\beta$-glucanasa y xilanasa

\begin{tabular}{|c|c|c|c|c|c|c|c|c|c|c|c|}
\hline 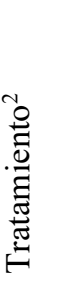 & 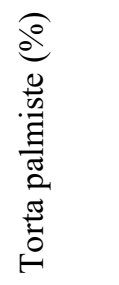 & 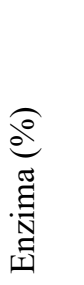 & 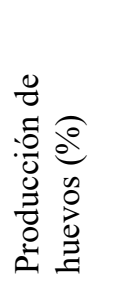 & $\begin{array}{l}\text { O0 } \\
0 \\
0 \\
0 \\
0 \\
0 \\
0 \\
0 \\
0 \\
0 \\
0\end{array}$ & 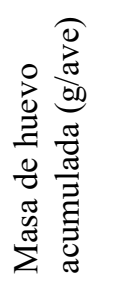 & 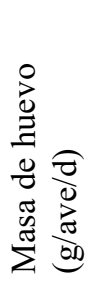 & 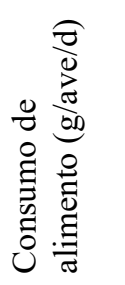 & 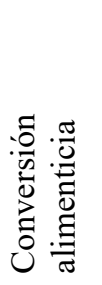 & 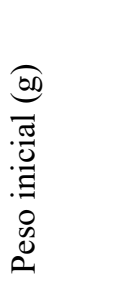 & 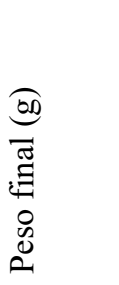 & 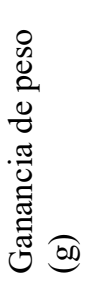 \\
\hline $\mathrm{T} 1$ & 0 & 0 & 81.31 & 66.33 & 4529.8 & 53.93 & 110.53 & 2.055 & 1994.3 & 2100.2 & 106.2 \\
\hline $\mathrm{T} 2$ & 0 & 0.05 & 79.10 & 66.44 & 4417.4 & 52.59 & 109.25 & 2.081 & 2002.3 & 2132.3 & 129.8 \\
\hline $\mathrm{T} 3$ & 3 & 0 & 80.39 & $68.50 *$ & 4624.9 & 55.06 & 112.03 & 2.038 & 2030.3 & 2215.7 & 186.5 \\
\hline $\mathrm{T} 4$ & 3 & 0.05 & 80.46 & 67.32 & 4549.6 & 54.16 & 110.62 & 2.042 & 2011.7 & 2168.2 & 156.5 \\
\hline T5 & 6 & 0 & 81.71 & 66.28 & 4549.2 & 54.16 & 110.32 & 2.040 & 1993.5 & 2144.3 & 150.7 \\
\hline T6 & 6 & 0.05 & 80.09 & 66.83 & 4483.6 & 53.38 & 110.79 & 2.083 & 1994.2 & 2140.5 & 146.3 \\
\hline \multirow{3}{*}{\multicolumn{2}{|c|}{$\begin{array}{c}\text { Efecto de la } \\
\text { torta de } \\
\text { palmiste }\end{array}$}} & 0 & 80.21 & 66.38 & 4473.6 & 53.26 & 109.89 & 2.068 & 1998.3 & 2116.2 & 118.0 \\
\hline & & 3 & 80.42 & 67.91 & 4587.1 & 54.61 & 111.32 & 2.040 & 2021.0 & 2191.9 & 171.0 \\
\hline & & 6 & 80.90 & 66.46 & 4516.4 & 53.77 & 110.55 & 2.062 & 1993.8 & 2142.4 & 148.5 \\
\hline \multirow{2}{*}{\multicolumn{2}{|c|}{$\begin{array}{l}\text { Efecto de las } \\
\text { enzimas }\end{array}$}} & 0 & 81.13 & 67.03 & 4567.9 & 54.38 & 110.96 & 2.044 & 2006.1 & 2153.4 & 147.4 \\
\hline & & 0.05 & 79.88 & 66.80 & 4483.5 & 53.37 & 110.21 & 2.069 & 2002.7 & 2147.0 & 144.2 \\
\hline \multicolumn{12}{|c|}{ Probabilidad } \\
\hline \multicolumn{3}{|c|}{ Piso de jaula } & 0.591 & 0.283 & 0.368 & 0.368 & 0.971 & 0.352 & 0.124 & 0.236 & 0.689 \\
\hline \multicolumn{3}{|c|}{$\begin{array}{l}\text { Niveles de torta } \\
\text { palmiste }(\mathrm{A})\end{array}$} & 0.918 & 0.001 & 0.463 & 0.464 & 0.731 & 0.678 & 0.370 & 0.146 & 0.217 \\
\hline \multicolumn{3}{|c|}{$\begin{array}{l}\text { Niveles de enzima } \\
\text { (B) }\end{array}$} & 0.456 & 0.594 & 0.411 & 0.411 & 0.617 & 0.579 & 0.979 & 0.970 & 0.953 \\
\hline \multicolumn{3}{|c|}{$\mathrm{A} \times \mathrm{B}$} & 0.850 & 0.196 & 0.983 & 0.983 & 0.839 & 0.875 & 0.872 & 0.658 & 0.688 \\
\hline
\end{tabular}

* Tratamiento difiere del control (0\%) por la prueba de Dunnett $(p<0.05)$

${ }^{1}$ Los valores de cada tratamiento representan el promedio de las seis repeticiones

2 T1: Dieta control; T2: Dieta con $0 \%$ torta de palmiste y $0.05 \% \beta$-glucanasa y xilanasa; T3: Dieta con $3 \%$ torta de palmiste y $0 \% \beta$-glucanasa y xilanasa; T4: Dieta con $3 \%$ torta de palmiste y $0.05 \% \beta$ glucanasa y xilanasa; T5: Dieta con $6 \%$ torta de palmiste y $0 \%$-glucanasa y xilanasa; T6: Dieta con 6 $\%$ torta de palmiste y $0.05 \%$-glucanasa y xilanasa

Los resultados obtenidos no concuerdan con los encontrados por Lawal et al. (2010), quienes reportan un menor peso final $(\mathrm{p}<0.05)$ en gallinas ponedoras alimentadas con dietas con alto contenido de fibra, sin suplementación de $\beta$-glucanasa + xilanasa; pero concuerdan con lo encontrado por Muñoz (2013), quien no encontró diferencias en la ganancia de peso de gallinas alimentadas con $6 \%$ de torta de palmiste con y sin enzimas ( $\beta$-glucanasa + xilanasa).
Consumo de alimento y conversión alimenticia

No hubo diferencias significativas para el consumo de alimento (g/ave/d) y la conversión alimenticia entre la dieta control con los demás tratamientos; lo cual indica que los niveles usados de torta de palmiste no afectan la palatabilidad del alimento ni su digestibilidad, tal y como ha sido reportado (Silversides et al., 2006; Muñoz, 2013). 
Cuadro 4. Consumo de nutrientes ${ }^{1}$ en gallinas ponedoras alimentadas con tres niveles de torta de palmiste y dos niveles de $\beta$-glucanasa y xilanasa

\begin{tabular}{|c|c|c|c|c|c|c|c|c|}
\hline \multicolumn{9}{|c|}{ Consumo de nutrientes (g) } \\
\hline 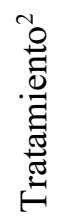 & 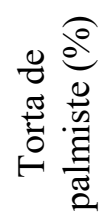 & 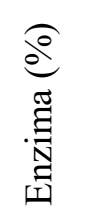 & 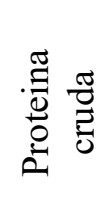 & $\sum_{I I}$ & 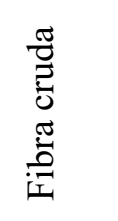 & 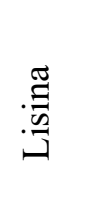 & 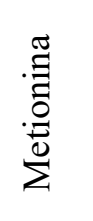 & 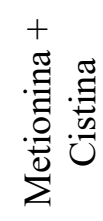 \\
\hline $\mathrm{T} 1$ & 0 & 0 & 16.26 & 3.106 & 2.84 & 0.86 & 0.46 & 0.75 \\
\hline $\mathrm{T} 2$ & 0 & 0.05 & 16.07 & 3.069 & 2.81 & 0.85 & 0.46 & 0.74 \\
\hline $\mathrm{T} 3$ & 3 & 0 & 16.47 & 3.170 & $3.37 *$ & 0.88 & 0.48 & 0.76 \\
\hline $\mathrm{T} 4$ & 3 & 0.05 & 16.26 & 3.130 & $3.33^{*}$ & 0.86 & 0.48 & 0.75 \\
\hline $\mathrm{T} 5$ & 6 & 0 & 16.22 & 3.099 & $3.91 *$ & 0.86 & 0.48 & 0.75 \\
\hline T6 & 6 & 0.05 & 16.29 & 3.114 & $3.92 *$ & 0.86 & 0.48 & 0.75 \\
\hline
\end{tabular}

* Tratamiento difiere del control (0\%) por la prueba de Dunnett $(p<0.05)$

${ }^{1}$ Los valores de cada tratamiento representan el promedio de las seis repeticiones

2 T1: Dieta control; T2: Dieta con $0 \%$ torta de palmiste y $0.05 \% \beta$-glucanasa y xilanasa; T3: Dieta con $3 \%$ torta de palmiste y $0 \% \beta$-glucanasa y xilanasa; T4: Dieta con $3 \%$ torta de palmiste y $0.05 \% \beta$ glucanasa y xilanasa; T5: Dieta con $6 \%$ torta de palmiste y $0 \% \beta$-glucanasa y xilanasa; T6: Dieta con 6 $\%$ torta de palmiste y $0.05 \% \beta$-glucanasa y xilanasa

Producción de huevos (\%), peso promedio de huevo (g), masa de huevo aситulada (g/ave) y masa de huevo (g/ave/d)

No se encontró diferencias significativas en la producción de huevos (\%) entre la dieta control con los demás tratamientos (Cuadro 3). En forma similar, Pérez et al. (2000) no encontraron diferencias significativas al utilizar niveles menores de $30 \%$ de torta de palmiste, al igual que Novak et al. (2008) usando torta de palmiste con la inclusión de un complejo enzimático (amilasa + xilanasa + proteasa).

El análisis de variancia para el peso promedio de huevo muestra diferencias significativas $(\mathrm{p}<0.05)$ a favor del tratamiento $\mathrm{T} 3$ con respecto al control (T1). El mayor peso de huevo posiblemente se deba al mayor peso de las aves (2215.67 vs. $2100.17 \mathrm{~g}$ en T3 y $\mathrm{T} 1$, respectivamente). Este resultado con- cuerda con lo encontrado por García et al. (2016), quienes al comparar el peso de las gallinas con el peso de los huevos, concluyeron que el peso corporal influye directa y positivamente sobre el peso del huevo.

La masa de huevo acumulada (g/ave) y la masa de huevo (g/ave/d/) no presentaron diferencias significativas entre tratamientos. Chong et al. (2008), asimismo, reportaron que las gallinas pueden tolerar hasta un $25 \%$ de torta de palmiste sin tener un impacto negativo sobre la producción de huevos o sobre la masa de huevos.

Consumo de proteina cruda, energía metabolizable y fibra cruda

No se encontraron diferencias significativas entre tratamientos con la dieta control en el consumo de proteína cruda y energía metabolizable; sin embargo, el consumo 
Cuadro 5. Calidad de huevo ${ }^{1}$ en gallinas ponedoras alimentadas con tres niveles de torta de palmiste y dos niveles de $\beta$-glucanasa y xilanasa

\begin{tabular}{|c|c|c|c|c|c|c|c|}
\hline Trat. $^{2}$ & $\begin{array}{c}\text { Torta } \\
\text { palmiste } \\
(\%)\end{array}$ & $\begin{array}{l}\text { Enzima } \\
(\%)\end{array}$ & $\begin{array}{l}\text { Pigmentación } \\
\text { (escala Roche) }\end{array}$ & $\begin{array}{c}\text { Espesor de } \\
\text { cáscara (mm) }\end{array}$ & $\begin{array}{c}\text { Altura de } \\
\text { clara } \\
(\mathrm{mm})\end{array}$ & $\begin{array}{c}\text { Altura de } \\
\text { yema } \\
(\mathrm{mm})\end{array}$ & $\begin{array}{c}\text { Unidades } \\
\text { Haugh }\end{array}$ \\
\hline $\mathrm{T} 1$ & 0 & 0 & 8.00 & 0.54 & 8.84 & 18.86 & 94.99 \\
\hline $\mathrm{T} 2$ & 0 & 0.05 & 7.67 & 0.54 & 9.10 & 19.48 & 96.87 \\
\hline $\mathrm{T} 3$ & 3 & 0 & 7.83 & 0.55 & 9.04 & 19.19 & 95.94 \\
\hline $\mathrm{T} 4$ & 3 & 0.05 & 7.83 & 0.51 & 9.17 & 19.01 & 96.76 \\
\hline $\mathrm{T} 5$ & 6 & 0 & 8.00 & 0.57 & 9.10 & 19.09 & 96.44 \\
\hline T6 & 6 & 0.05 & 7.83 & 0.52 & 8.72 & 19.05 & 94.73 \\
\hline \multirow{3}{*}{\multicolumn{2}{|c|}{$\begin{array}{l}\text { Efecto de la torta de } \\
\text { palmiste }\end{array}$}} & 0 & 7.83 & 0.54 & 8.97 & 19.17 & 95.93 \\
\hline & & 3 & 7.83 & 0.53 & 9.10 & 19.10 & 96.35 \\
\hline & & 6 & 7.91 & 0.54 & 8.91 & 19.05 & 95.58 \\
\hline \multirow{3}{*}{\multicolumn{2}{|c|}{$\begin{array}{l}\text { Efecto de las } \\
\text { enzimas }\end{array}$}} & 0 & 7.94 & 0.55 & 9.15 & 19.05 & 95.79 \\
\hline & & 0.05 & 7.78 & 0.52 & 9.05 & 19.16 & 96.12 \\
\hline & & & & Probabilidad & & & \\
\hline \multicolumn{3}{|c|}{ Piso de jaula } & 0.747 & 0.459 & 0.924 & 0.050 & 0.865 \\
\hline \multicolumn{3}{|c|}{ Niveles de torta palmiste (A) } & 0.822 & 0.751 & 0.779 & 0.476 & 0.865 \\
\hline \multicolumn{3}{|c|}{ Niveles de enzima (B) } & 0.170 & 0.065 & 0.993 & 0.579 & 0.799 \\
\hline \multicolumn{3}{|c|}{$\mathrm{A} \times \mathrm{B}$} & 0.538 & 0.368 & 0.479 & 0.076 & 0.437 \\
\hline
\end{tabular}

* Tratamiento difiere del control (0\%) por la prueba de Dunnett $(p<0.05)$

${ }^{1}$ Los valores de cada tratamiento representan el promedio de las seis repeticiones

${ }^{2}$ T1: Dieta control; T2: Dieta con $0 \%$ torta de palmiste y $0.05 \% \beta$-glucanasa y xilanasa; T3: Dieta con $3 \%$ torta de palmiste y $0 \% \beta$-glucanasa y xilanasa; T4: Dieta con $3 \%$ torta de palmiste y $0.05 \% \beta$ glucanasa y xilanasa; T5: Dieta con $6 \%$ torta de palmiste y $0 \%$-glucanasa y xilanasa; T6: Dieta con 6 $\%$ torta de palmiste y $0.05 \%$-glucanasa y xilanasa

de fibra fue significativamente mayor en T3T6 en comparación con T1 y T2 $(\mathrm{p}<0.05)$ (Cuadro 4).

\section{Consumo de lisina, metionina y metionina + cistina}

No hubo diferencias estadísticas significativas entre la dieta control con los demás tratamientos en el consumo de lisina, metionina y metionina + cistina (Cuadro 4), indicando que las dietas tuvieron los niveles de aminoácidos adecuados para asegurar el consumo de alimento y, consecuentemente, el consumo de lisina y de metionina + cistina. En gallinas ponedoras, la metionina es el pri- mer aminoácido limitante, seguido de la lisina en dietas de maíz-soya (Schutte y De Jong, 1998), teniendo gran influencia sobre el peso de huevo y la producción. Esto queda corroborado con los resultados obtenidos para $\mathrm{T} 3$ al maximizar la producción de huevo (Cuadro 3).

\section{Calidad de Huevo}

Pigmentación de la yema

La pigmentación de la yema en todos los tratamientos, de acuerdo con la escala de Roche, estuvo entre 7 a 8, coloración amarilla-clara, sin diferencias significativas entre 
tratamientos (Cuadro 5); posiblemente debido a que todas las dietas tuvieron prácticamente la misma cantidad de maíz (60\%), que aporta carotenoides amarillos (zeaxantina). Además, en todas las dietas se incorporó $0.02 \%$ de pigmentante (extracto de Marigold).

\section{Espesor de cáscara}

No hubo diferencias significativas entre la dieta control con los demás tratamientos en el espesor de cáscara de huevo (Cuadro 5), lo cual concuerda con Chong et al. (2008) donde el espesor de la cáscara de huevo no fue afectado con niveles de hasta $25 \%$ de torta de palmiste. Asimismo, Novak et al. (2008) no encontraron efectos de enzimas exógenas sobre el espesor de la cáscara de huevo de ponedoras comerciales.

Altura de la clara y de la yema

No hubo diferencias significativas entre la dieta control con los demás tratamientos en la altura de la clara y la altura de yema (Cuadro 5). La altura de la clara fue entre 8.72 y $9.17 \mathrm{~mm}$, lo que indica frescura de los huevos (fueron evaluados en mismo día de la puesta) y que las dietas suministradas cubrieron satisfactoriamente los aminoácidos esenciales requerido por las aves. Asimismo, la altura de la yema (18.86-19.48 mm) fue adecuada, lo que evidencia que las dietas usadas cubrieron los requerimientos de grasa y demás nutrientes.

\section{Unidades Haugh}

No se encontró diferencias significativas entre la dieta control con los demás tratamientos en Unidades Haugh (Cuadro 5). Esta respuesta era de esperar; ya que las Unidades Haugh, medida de la calidad proteica del huevo, correlacionan la altura de la clara con el peso del huevo, parámetros en los cuales tampoco se encontraron diferencias. Los valores en todos los tratamientos fueron superiores a 90 (calidad excelente), debido en parte a la frescura del huevo. Este resultado concuerdo con los estudios de Chan-
Colli et al. (2007) y Oliveira et al. (2009), quienes manifiestan que la disminución del valor de esta variable está influenciada por el tiempo y el método de conservación.

\section{Conclusiones}

La adición de hasta $6.5 \%$ de torta de palmiste en reemplazo parcial del maíz, con y sin la adición de las enzimas $\beta$-glucanasa y xilanasa en un $0.05 \%$, no afecta el rendimiento productivo ni la calidad del huevo en gallinas ponedoras.

\section{Literatura Citada}

1. Chan-Colli M, Carvajal M, Segura J, Sarmiento L, Santos R. 2007. Effect of dietary energy and sulphur amino acid level on egg production traits in the tropics. J Anim Vet Adv 6: 1209-1213.

2. Chong CH, Zulkifli I, Blair R. 2008. Effects of dietary inclusion of palm kernel cake and palm oil, and enzyme supplementation on performance of laying hens. Asian Austral J Anim 21: 1053-1058. 10.5713/ajas.2008.70581

3. DSM. Bright Science Brighter Living. 2013. Guía de DSM para pigmentación de la yema de huevo con Carophyll. [Internet]. Disponible en: https:// www.dsm.com/content/dam/dsm/anh/ en_US/documents/carophyll_guidelines_amended_SPAN_web.pdf

4. [FEDNA] Fundación Española para el Desarrollo de la Nutrición Animal. 2015. Tablas de composición y valor nutritivo de alimentos para la fabricación de piensos compuestos. $3^{\mathrm{a}}$ ed. [Internet]. Disponible en: $\underline{\text { http:}: / / f u n d a c i o n f e d n a . o r g / ~}$ node/439

5. García DM, Colas MC, López WS, Pérez EOR, Sánchez AP, Lamazares MCP, Grandía RG 2016. El peso corporal y su efecto sobre indicadores bioproductivos en gallinas White Leghorn L33. Rev Med Vet Zoot 63: 188-200. 
6. Lawal TE, Iyayi EA, Adeniyi BA, Adaromoye $O A$. 2010. Biodegradation of palm kernel cake with multienzymes complexes from fungi and its feeding value for broilers. Int J Poultry Sci 9: 695-701.

7. [MINAGRI] Ministerio de Agricultura y Riego. 2016. Plan Nacional de Desarrollo Sostenible de la Palma Aceitera en el Perú (2016-2025). [Internet]. Disponible en: http: //repositório.minagri.gob.pe/handle/MINAGRI/29

8. [MINAGRI] Ministerio de Agricultura y Riego. 2018. Importación de maíz amarillo duro aumentó $9.8 \%$ durante el 2017. [Internet]. Disponible en: http:// www.agraria.pe/noticias/importacionde-maiz-amarillo-duro-aumento-98-durante-el-2017-15710

9. Muñoz C. 2013. La torta de palmiste más enzimas exógenas en la alimentación de ponedoras comerciales. Tesis de Ingeniero Zootecnista. Riobamba, Ecuador: Escuela Superior Politécnica de Chimborazo. $84 \mathrm{p}$.

10. Novak CL, Yakout HM, Remus J. 2008. Response to varying dietary energy and protein with or without enzyme supplementation on Leghorn performance and economics. 2. Laying period. J Appl Poultry Res 17: 17-33. doi: 10.3382/japr.2006-00126

11. NRC. 1994. Nutrient requirements of poultry. $9^{\text {th }}$ rev ed. Washington DC: National Academy Press. 155 p.

12. Oliveira GE, Figueiredo TC, Souza MR, Oliveira AL, Cançado SV, Gloria $M B$. 2009. Bioactive amines and quality of egg from Dekalb hens under different storage conditions. Poultry Sci 88: 24282434. doi: 10.3382/ps.2009-00028

13. Perez JF, Gernat AG, Murillo JG. 2000. The effect of different levels of palm kernel meal in layer diets. Poultry Sci 79: 77-79. doi: 10.1093/ps/79.1.77

14. SAS Statistical Analysis System. 2009. User's Guide. Version 9.2 ed. SAS Inst. Inc., Cary, NC.

15. Schutte JB, De Jong J. 1998. Ideal amino acid profile for poultry. Options Mediterranéennes 9: 259-263.

16. Silversides FG, Scott TA, Korver DR, Afsharmanesh M, Hruby M. 2006. Study on the interaction of xylanase and phytase enzymes in wheat-based diets fed to commercial white and brown egg laying hens. Poultry Sci 85: 297-305. doi: 10.1093/ps/85.2.297 